\section{Y-12}

OAK RIDGE

\section{Y-12 \\ PLANT}

Report No.: Y/LB-16,012

\title{
PASSIVE NMIS MEASUREMENTS TO ESTIMATE THE SHAPE OF PLUTONIUM ASSEMBLIES
}

\author{
J. K. Mattingly \\ J. A. March-Leuba \\ J. T. Mihalczo \\ R. B. Perez \\ L. G. Chiang \\ T. E. Valentine \\ J. A. Mullens
}

Nuclear Materials Management and Storage Program Office

July 22, 1999

MANAGED BY

LOCKHEED MARTIN ENERGY SYSTEMS, INC. FOR THE UNITED STATES

DEPARTMENT OF ENERGY
Prepared by the Oak Ridge Y-12 Plant

Oak Ridge, Tennessee 37831 managed by

Lockheed Martin Energy Systems, Inc. for the U.S. DEPARTMENT OF ENERGY under contract DE-AC05-84OR21400 


\section{DISCLAIMER}

This report was prepared as an account of work sponsored by an agency of the United States government. Neither the United States Government nor any agency thereof, nor any of their employees, makes any warranty, express or implied, or assumes any legal liability or responsibility for the accuracy, completeness, or usefulness of any information, apparatus, product, or process disclosed, or represents that its use would not infringe privately owned rights. Reference herein to any specific commercial product, process, or service by trade name, trademark, manufacturer, or otherwise, does not necessarily constitute or imply its endorsement, recommendation, or favoring by the United States Government or any agency thereof. The views and opinions of authors expressed herein do not necessarily state or reflect those of the United States Government or any agency thereof.

\section{COPYRIGHT NOTICE}

The submitted manuscript has been authored by a contractor of the U.S. Government under contract DE-AC05-840R21400. Accordingly, the U.S. Government retains a paid-up, nonexclusive, irrevocable, worldwide license to publish or reproduce the published form of this contribution, prepare derivative works, distribute copies to the public, and perform publicly and display publicly, or allow others to do so, for U.S. Government purposes. 


\section{DISCLAIMER}

Portions of this document may be illegible in electronic image products. Images are produced from the best available original document. 


\title{
PASSIVE NMIS MEASUREMENTS TO ESTIMATE THE SHAPE OF PLUTONIUM ASSEMBLIES
}

\author{
J. K. Mattingly, J. A. March-Leuba, J. T. Mihalczo, R. B. Perez, L. G. Chiang, \\ T. E. Valentine, J. A. Mullens \\ Instrumentation and Controls Division, Oak Ridge National Laboratory \\ P.O. Box 2008 MS6004, Oak Ridge, TN 37831-6004
}

\begin{abstract}
A new technique to estimate the shape attribute of plutonium assemblies using the Nuclear Materials Identification System (NMIS) is described. The proposed method possesses a number of advantages. It is passive: no external radiation source is required to estimate the shape of plutonium assemblies. Instead, inherent gamma and neutron emissions from spontaneous fission of ${ }^{240} \mathrm{Pu}$ and subsequent induced fission of ${ }^{239} \mathrm{Pu}$ are detected to estimate the shape attribute. The technique is also stationary: shape is estimated without scanning the assembly by moving the detectors relative to the assembly.
\end{abstract}

The proposed method measures third order correlations between triplets of gamma/neutron-sensitive detectors. The real coincidence of a pair of gammas is used as a "trigger" to approximately identify the time of a spontaneous or induced fission event. The spatial location of this fission event is inferred from the real coincidence of a subsequent neutron with the initial pair of correlated gammas by using the neutron's time-of-flight (approximately the delay between the gamma pair and the neutron) and the fission neutron spectra of ${ }^{240} \mathrm{Pu}$ and ${ }^{239} \mathrm{Pu}$. The spatial distribution of fission sites and hence the approximate shape of the plutonium assembly is thereby inferred by measuring the distribution of a large number of these correlated triplets.

Proof-of-principle measurements were performed using ${ }^{252} \mathrm{Cf}$ as a surrogate for ${ }^{240} \mathrm{Pu}$ to demonstrate that the technique is feasible. For the simple shapes approximated with ${ }^{252} \mathrm{Cf}$ sources, the measurements showed that the proposed method is capable of correctly identifying the shape and accurately estimating its size to within a few percent of actual.

\section{Introduction}

Both domestic and international safeguards efforts, particularly as they pertain to the protection of nuclear weapons and their components, could benefit from the development of a technique to passively estimate the shape and size of containerized plutonium assemblies. Especially for cooperative international agreements, e.g., START, the technique developed should be capable of characterizing the shape attribute without using prototypical template measurements, because these may contain potentially sensitive information and therefore typically cannot be shared between the parties of the agreement. Furthermore, for domestic safeguards, current measurement techniques that do perform comparisons to prototypical templates often suffer from initialization problems. Many of these techniques lack a method to independently verify that the identity of the initial template item is indeed valid for subsequent comparisons, i.e., that the template item is in fact a weapon or component. However, a technique that could accurately estimate the shape and size of containerized plutonium assemblies without using template comparison could also be used to subsequently validate template items and thereby solve at least part of this template initialization problem.

The method proposed in this paper could be developed into the desired technology. It is a technique that can potentially identify containerized regular shapes of plutonium without using any template for comparison. The method proposed uses the Nuclear Materials Identification System (NMIS) operating in passive mode, i.e., without any active source, to estimate the shape of plutonium assemblies by detecting their spontaneous neutron and gamma emissions. Furthermore, the proposed technique is stationary, i.e., the assembly is not scanned by moving the detectors relative to the assembly to estimate the shape. This feature is particularly desirable since a serious concern associated with any scanning technique is that observation of the scanning process could reveal sensitive information, e.g., locations and sizes. A stationary measurement does not suffer from this weakness.

Subsequently, the fundamental features of the proposed technique and its implementation are described. A demonstrative implementation of the method was developed to test its feasibility. Because plutonium was not readily 
available, californium was used as a surrogate to construct approximate simply-shaped distributed sources of spontaneous fission neutrons and gammas. The configuration and results of these proof-of-principle experiments are described and are used to draw conclusions regarding the potential capabilities of the proposed method.

\section{Fundamental Features of Proposed Method}

Weapons-grade plutonium typically contains a substantial fraction of ${ }^{240} \mathrm{Pu}$, a spontaneous fission source of neutrons and gammas. If it is assumed (as is reasonable) that the ${ }^{240} \mathrm{Pu}$ is uniformly dispersed throughout the plutonium, then the spatial distribution of spontaneous fission sites is congruent to the shape of the assembly. Hence, estimating the distribution of spontaneous fission sites is tantamount to estimating the shape.

The proposed method infers the spatial distribution of fission sites using an array of gamma/neutron-sensitive detectors to acquire simultaneous estimates of the distribution of distances from the fission sites to each detector in the array. In concept, the method is (somewhat) analogous to the technique used by the global positioning system (GPS) to locate a point in three-dimensional space. ${ }^{1}$ The concept is illustrated in Fig. 1 for a point radiation source in a plane defined by three detectors, i.e., for the two-dimensional case. An estimate of the distance $\left(R_{1}, R_{2}\right.$, or $R_{3}$ in the figure) between the point source and a given detector defines a circle about that detector. The source must lie at the point common to the three circles surrounding the detectors. In the three-dimensional case, each distance estimate defines a sphere around the corresponding detector such that four detectors must be used to locate the source at the unique point common to the four spheres surrounding the detectors.

To understand the generalization of the technique from one that measures the location of a point source to one that measures the shape of a spatially distributed source, recognize that any distributed source is simply a collection of point sources. For example, a sphere (by definition) is the locus of points within the volume defined by the sphere's radius and center.

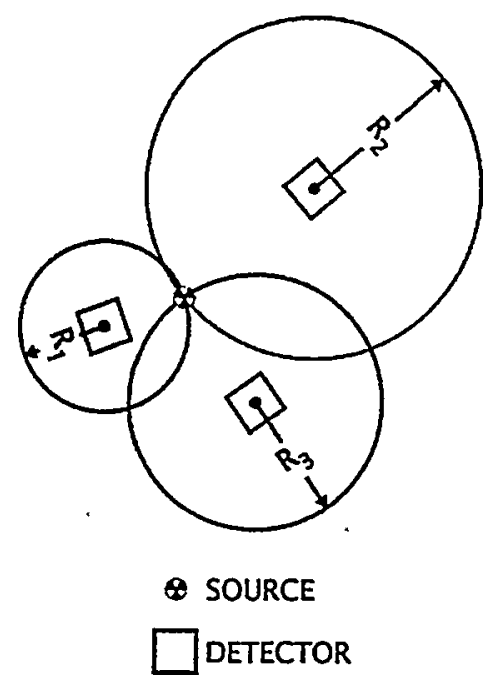

Figure 1. The method to estimate the location of a point source

In order to measure the shape of a distributed source, it is necessary to estimate the distribution of distances from points within the source volume to each detector in the array. The distributions of distances can then be used to estimate the distribution of fission sites and hence the shape of the plutonium assembly.

\section{Proposed Implementation of Method}

Consider that the time it takes a neutron to "fly" from a spontaneous fission to a detector is simply the distance from the fission site to the detector divided by the neutron's flight-speed. Consequently, the distribution of neutron flighttimes following fission, i.e., the neutron time-of-flight spectrum, is congruent to the distribution of flight-speeds (the fission spectrum) convolved with the distribution of flight-distances. Hence the distribution of flight-distances can be deconvolved from the time-of-flight spectrum if a fission spectrum is assumed.

Recall that spontaneous (and induced fission) events may release multiple gammas (and neutrons). Note that because all gammas travel at the speed of light, the real coincidence of near-simultaneous gammas approximately marks the time of fission. Because gammas travel at roughly $300 \mathrm{~mm} / \mathrm{nsec}$, a coincidence window of only a few nanoseconds can measure simultaneity between gammas counted in two detectors separated by a much as a meter. Furthermore, because fission neutrons travel at much slower speeds (on the order of tens of $\mathrm{mm} / \mathrm{nsec}$ ), relative to typical neutron flight-times the near-simultaneous gamma pair can also be regarded as near-instantaneous following the fission event. Hence, a

\footnotetext{
'The analogy is actually in reverse; GPS satellites emit time-stamped signals that a GPS receiver uses to estimate location.[1,2] For the method to locate a point radiation source, the source is the emitter and each detector acts as a receiver. The analogy lies in both methods' use of intersecting spheres to locate a unique point in space.
} 
simultaneous gamma pair can be used as an approximate "trigger" to measure neutron time-of-flight. Consequently, the time-distribution of neutron counts following simultaneous gamma pairs can be used to estimate the neutron timeof-flight spectrum.

There are a number of means to implement a technique to measure the time-distribution of neutron counts following simultaneous gamma pairs. The proposed method uses third-order correlation techniques to measure the distribution of three-way coincidence over the relative time-delays between triplets of detector channels.

The third-order coincidence distribution

$$
C_{123}\left(\tau_{12}, \tau_{13}\right)=\left.\overline{x_{1}\left(t_{1}\right) x_{2}\left(t_{2}\right) x_{3}\left(t_{3}\right)}\right|_{\tau_{12}=t_{2}-t_{1}}
$$

is the distribution of three-way coincident (bicoincident) detection events over the delay $\tau_{12}=t_{2}-t_{1}$ between the event $x_{1}\left(t_{1}\right)$ in the first channel and the event $x_{2}\left(t_{2}\right)$ in the second channel and the delay $\tau_{13}=t_{3}-t_{1}$ between the event $x_{1}\left(t_{1}\right)$ in the first channel and the event $x_{3}\left(t_{3}\right)$ in the third channel.[3] As in the case of the second-order distribution of events coincident at a relative delay of $\tau$,

$$
C_{12}(\tau)=\left.\overline{x_{1}\left(t_{1}\right) x_{2}\left(t_{2}\right)}\right|_{\tau=t_{2}-t_{1}},
$$

some coincidences are "real," i.e., are correlated, and some coincidences are "accidental," i.e., are uncorrelated. So, analogous to the covariance

$$
R_{12}(\tau)=C_{12}(\tau)-\overline{x_{1}} \overline{x_{2}}
$$

that measures the distribution of real two-way coincidence over the delay $\tau$ between channels, the bicovariance

$$
R_{123}\left(\tau_{12}, \tau_{13}\right)=C_{123}\left(\tau_{12}, \tau_{13}\right)-\overline{x_{1}} R_{23}\left(\tau_{13}-\tau_{12}\right)-\overline{x_{2}} R_{13}\left(\tau_{13}\right)-\overline{x_{3}} R_{12}\left(\tau_{12}\right)-\overline{x_{1}} \overline{x_{2}} \overline{x_{3}},
$$

measures the distribution of real three-way coincidence over the respective delays $\tau_{12}$ and $\tau_{13}$ between channels 1 and 2 and between channels 1 and 3 .

When the bicovariance is measured from a spontaneous fission source using detectors that are sensitive to both neutrons and gammas, the following four kinds of real bicoincident triplets will be observed.

- Gamma-gamma-gamma triplets (G-G-G): three gammas from a single spontaneous fission.

- Each count in a G-G-G triplet will tend to occur in rapid succession because each gamma moves at the speed of light.

- Hence the time-delays between individual counts in the triplet will be very short (a few nanoseconds).

- Consequently, G-G-G triplets tend to appear as a concentrated "peak" about the $\left(\tau_{12}, \tau_{13}\right)$-origin.

- Gamma-gamma-neutron triplets (G-G-N): two gammas and a neutron from a single spontaneous fission.

- The two gammas will always be counted first in sequence and the neutron will tend to be counted much later according to its time-of-flight, i.e., the distance from the spontaneous fission site to the detector divided by the neutron's flight-speed.

- Hence the time-delay between the two gamma counts will be very short (a few nanoseconds) and the time-delay following the gamma count pair to the neutron count will be distributed (over tens of nanoseconds) according to the spontaneous fission prompt neutron spectrum.

- Consequently G-G-N triplets appear as "ridges" concentrated along the $\tau_{12}$-and $\tau_{13}$-axes and along the diagonal $\tau_{12}=\tau_{13} \leq 0$; the shape of each ridge is congruent to the neutron time-of-flight spectrum.

- Gamma-neutron-neutron triplets (G-N-N): one gamma and two neutrons from a single spontaneous fission.

- The gamma will always be counted first in sequence, and the individual neutrons in the subsequent pair will tend to be counted much later according to their individual times-of-flight.

- Hence the time-delay between the initial gamma count and each subsequent neutron count will tend to be much greater than zero (tens of nanoseconds). 
- Consequently G-N-N triplets appear as "lobes" between the G-G-N ridges; the shape of each lobe is congruent to the neutron time-of-flight spectrum convolved once with itself.

- Neutron-neutron-neutron (N-N-N): three neutrons from a single spontaneous fission.

- Each neutron in the triplet will be counted according to its time-of-flight from the spontaneous fission site to the detectors.

- Hence the time-delays between each neutron count will distributed according to the difference in the flight-speed of each neutron in the $\mathrm{N}-\mathrm{N}-\mathrm{N}$ triplet.

- Consequently N-N-N triplets tend to appear as a broad "bump" centered near the origin with a shape congruent to the neutron time-of-flight spectrum convolved twice with itself.

These various "features" are illustrated in Fig. 2 which shows the bicovariance passively measured from a ${ }^{252} \mathrm{Cf}$ source using three plastic scintillators (arranged as depicted in the graphic). Recognize that the relative amplitude of each feature is controlled by the detectors' respective efficiencies for counting neutrons and gammas and by the relative probabilities of prompt neutron and gamma emission from spontaneous fission of ${ }^{252} \mathrm{Cf}$. Consequently, despite the fact that these detectors have much higher neutron efficiencies than gamma efficiencies, the distribution of N-N$\mathrm{N}$ triplets is obscured by the more prominent distributions of triplets involving one or more gammas because ${ }^{252} \mathrm{Cf}$ has a greater propensity for emitting prompt fission gammas (average 7.8 per spontaneous fission) than for emitting prompt fission neutrons (average 3.8 per spontaneous fission).

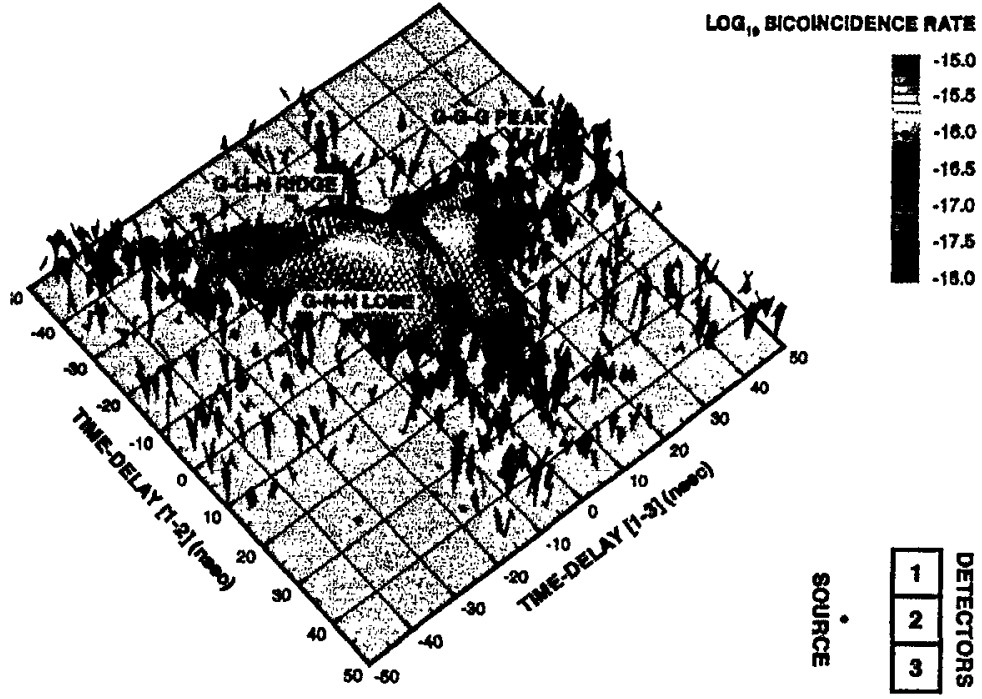

Figure 2. The bicovariance measured from a ${ }^{252} \mathrm{Cf}$ source using three plastic scintillators arranged as shown in the graphic at lower right; the bicoincidence rate has units of real bicoincident triplets/nsec ${ }^{3}$

As noted in Fig. 3, each of the three G-G-N ridges measures the time-distribution of neutron counts in the noted detector correlated with the real coincidence of a near-simultaneous pair of gamma counts in the other two detectors. - Along the $\tau_{12}$-axis, the near-simultaneous gamma pair "trigger" is counted by detector pair $(1,2)$ and the subsequent neutron is counted by detector 3 . Hence, G-G-N ridge 3 approximately measures the neutron time-of-flight spectrum observed by detector 3 .

- Along the $\tau_{13}$-axis, the near-simultaneous gamma pair trigger is counted by detector pair $(1,3)$ and the subsequent neutron is counted by detector 2 . Hence, G-G-N ridge 2 approximately measures the neutron time-of-flight spectrum observed by detector 2 .

- Along the diagonal $\tau_{12}=\tau_{13} \leq 0$, the near-simultaneous gamma pair trigger is counted by detector pair $(2,3)$ and the subsequent neutron is counted by detector 1 . Hence, G-G-N ridge 1 approximately measures the neutron time-offlight spectrum observed by detector 1 .

Further observe that each ridge is "dilated" according to the distance between the source and the corresponding detector.

This dilation occurs for the following reasons. Prompt neutrons emerge from the spontaneous fission source with kinetic energy according to the source's spectrum, and neutrons incident on a given detector are counted according to its' neutron detection efficiency. Hence, the probability of counting a prompt fission neutron incident on the detector is simply the product of the fission spectrum and the detection efficiency; these are both dependent upon the neutron's flight-speed. Because, for a given flight-distance, the neutron flight-time from the source to the detector is simply the distance divided by the speed, the time-of-flight spectrum of detected neutrons is dilated by the flight-distance. 


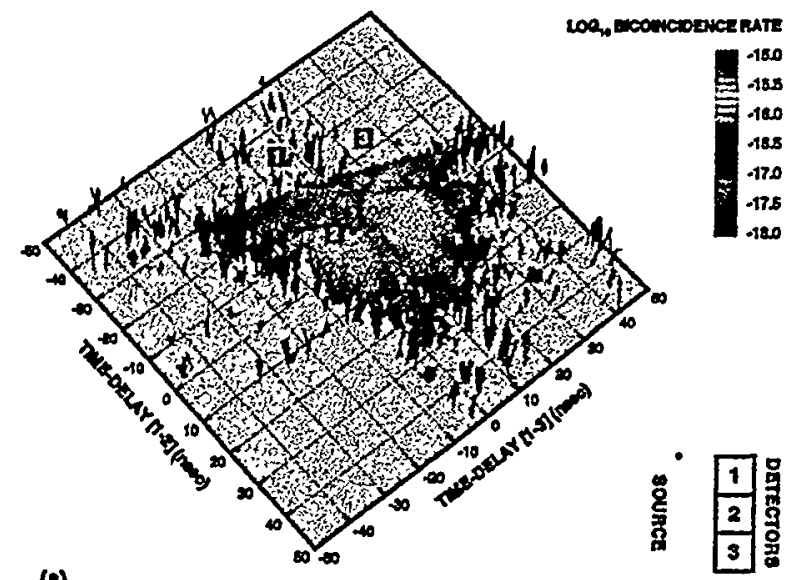

(a)

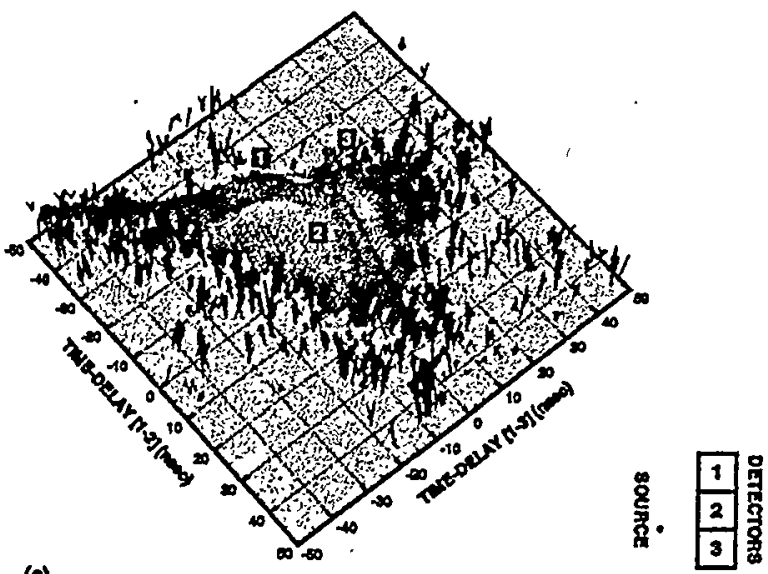

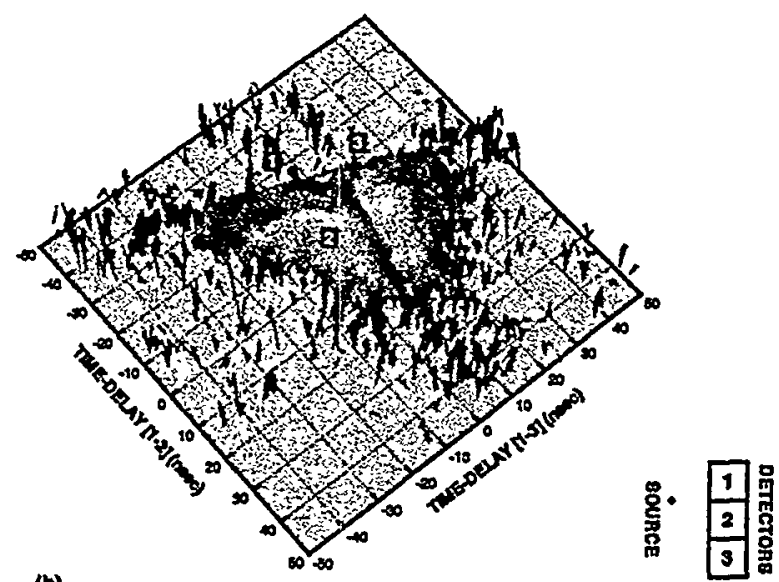

(b)

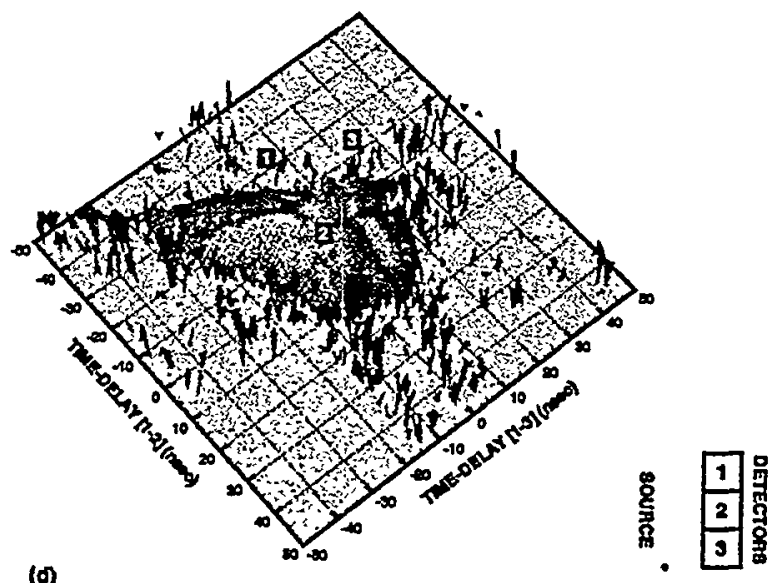

Figure 3. Each gamma-gamma-neutron (G-G-N) ridge in the bicovariance is dilated according to the distance from the source to the corresponding detector

Consequently, for an assumed spontaneous fission prompt neutron spectrum and known neutron detection efficiency, the time-of-flight spectrum provides a means to passively measure the distance from the detector back to the original spontaneous fission site. ${ }^{2}$

Consequently the bicovariance between each triplet of detectors provides a simultaneous measurement of the distance from the source to each detector in the triplet. As the following proof-of-principle measurements show, this allows the absolute location of a point source to be estimated and the shape and size of a distributed source to be estimated. Because plutonium was unavailable, ${ }^{252} \mathrm{Cf}$ was used as a surrogate spontaneous fission source of neutrons and gammas.

\section{Demonstration of Feasibility of Implementation}

The first series of measurements, illustrated in Fig. 4, were performed to test the technique's ability to locate a point source in two-dimensional $(x, y)$-coordinates. The point source shown in Fig. 4 was moved to moved to seven different locations relative to the array of three plastic scintillators.

${ }^{2}$ To a first-order approximation, the prompt neutron spectra of most spontaneously fissioning and fissionable isotopes are similar enough to yield time-of-flight spectra that are roughly equivalent. [4] Furthermore, the neutron detection efficiency of proton-recoil detectors can be accurately modeled as $s$-wave neutron scattering off hydrogen nuclei and can be precisely measured from the time-of-flight spectrum using a ${ }^{252} \mathrm{Cf}$ ionization chamber as a point neutron source with a known spectrum. $[5,6]$ 


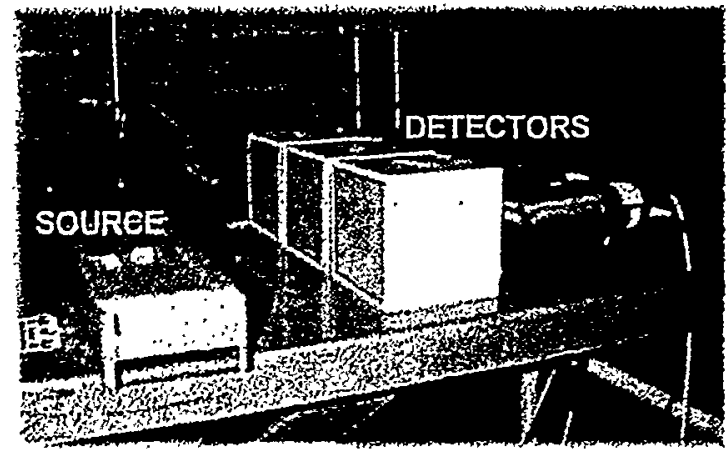

Figure 4. Measurements performed to estimate the location of a point source; the ${ }^{252} \mathrm{Cf}$ source was moved to seven different locations relative to detector array

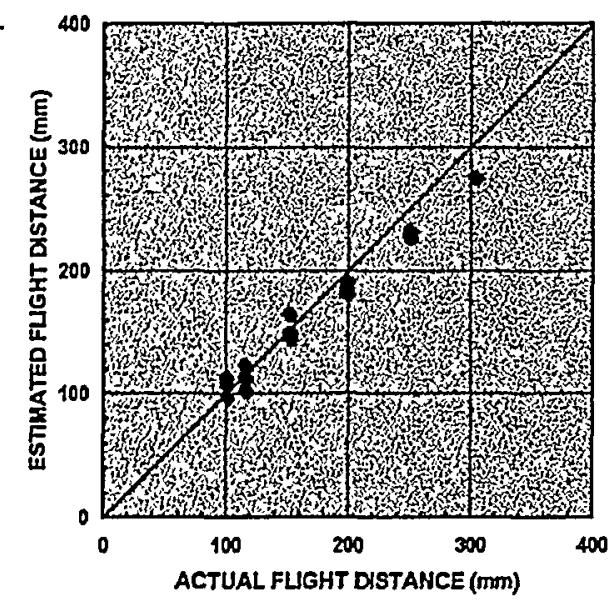

Figure 5. Estimated distances from the source to each detector versus actual distances

The series of measurements illustrated in Fig. 7 were performed to test the technique's ability to distinguish between a point source and a distributed source and to estimate the size of a distributed source. It was not possible to obtain a continuously distributed source, so ring sources of spontaneous fission gammas and neutrons were approximated by distributing $s^{2}{ }^{252} \mathrm{Cf}$ sources around three circles. As shown, the detectors were arranged on a circle with the diameter of an AT400-R container, and the sources were distributed on circles with diameters of $10 \mathrm{~mm}$ (an approximate point source), 160 $\mathrm{mm}(1 / 3$ the diameter of an AT400-R), and $320 \mathrm{~mm} \mathrm{(2/3}$ AT400-R diameter).[7]

Figure 8 depicts the bicovariance measured from each approximate ring source. Note that the G-G-N ridges in the
Figure 5 shows the resulting twenty-one estimates of flightdistance (one distance to each of three detectors at seven source locations) compared to the actual flight-distance. All distance estimates were within $-27 \mathrm{~mm}$ to $+13 \mathrm{~mm}$ of the actual flight-distance such that the relative error in each estimate was less than $11 \%$.

The three simultaneous estimates of flight-distance at each of the seven source positions were used to solve for the source location in $(x, y)$-coordinates, and the resulting estimates of source location are compared to the actual location in Fig. 6. In the figure, the detector array is located at the $x$-origin. Observe that the greatest error in the estimated source location is $33 \mathrm{~mm}$ (root-mean-square distance). Consequently, the technique is capable of locating a point source. The subsequent proof-of-principle measurements were performed to implement the generalization of the method to estimate the shape and size of a distributed source.

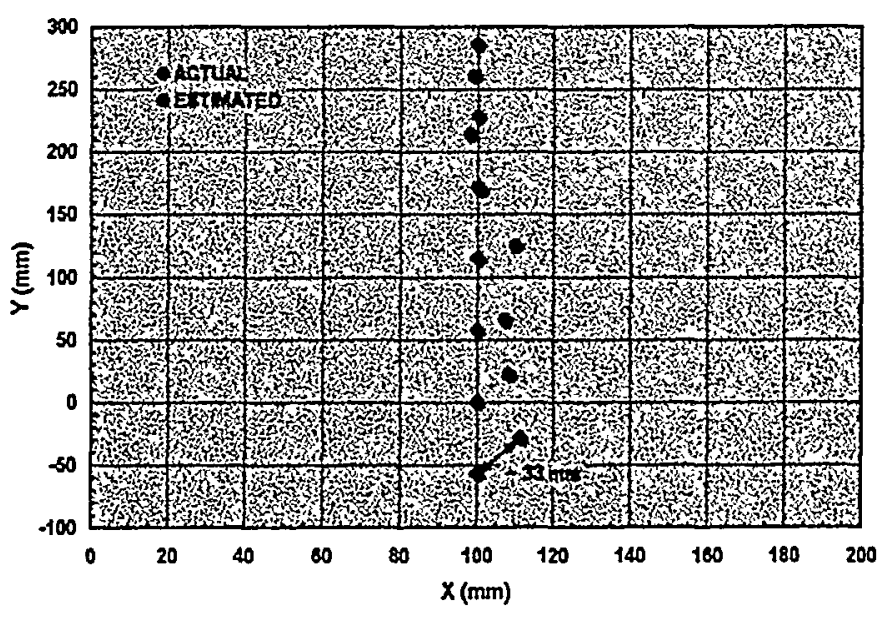

Figure 6. Estimated locations of the source versus actual locations

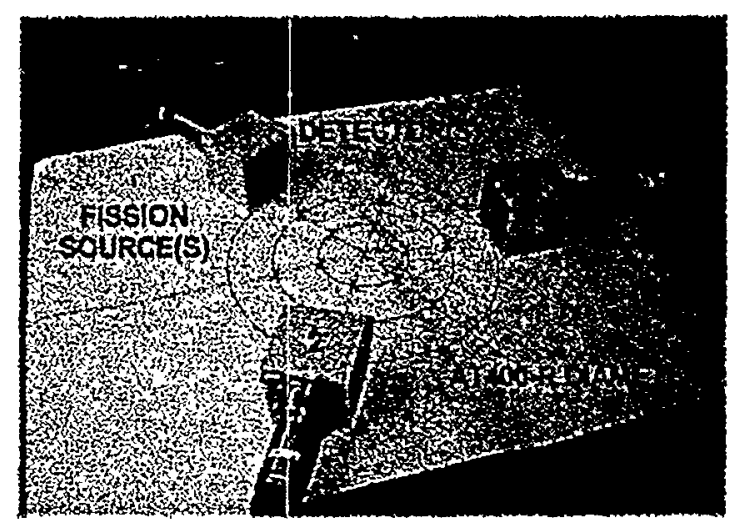

Figure 7. Measurements performed to estimate the shape and size of approximate ring sources; $\operatorname{six}^{252} \mathrm{Cf}$ sources (the $X^{\prime} \mathrm{s}$ ) were distributed at a point and $1 / 3$ and $2 / 3$ the diameter of an AT400-R container 


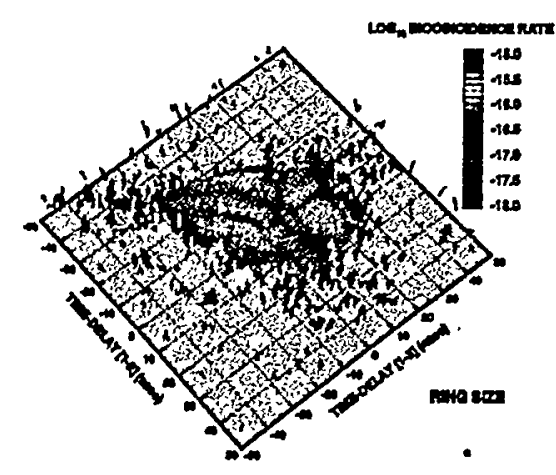

(a)

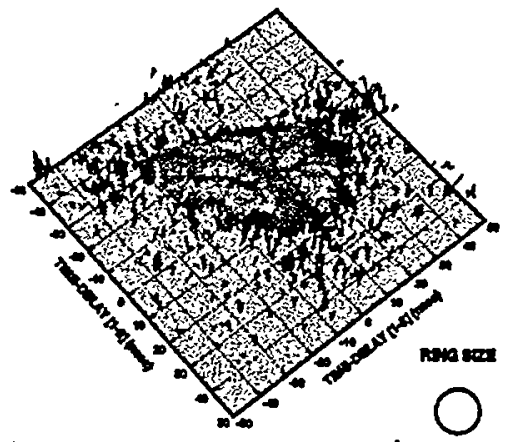

(1)

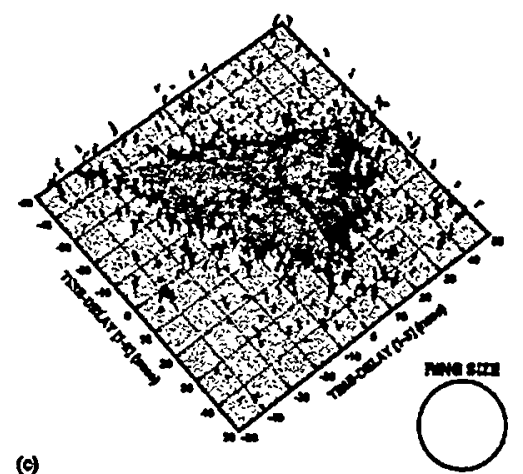

Figure 8. The bicovariance measured from each approximate ring source

bicovariance measured from the 160-mm-diameter and 320-mm-diameter sources do not correspond to the time-offlight spectrum expected from a point fission source. Consequently, the technique can readily distinguish between point and distributed sources.

Figure 9 compares the diameter estimated for each ring source to its actual diameter., Observe that the diameter estimated for the point configuration is approximately zero and that the diameters estimated for the 160 -mm- and $320-\mathrm{mm}$-rings deviate from the actual by less than $10 \mathrm{~mm}$. Therefore, the technique appears to be able to accurately estimate the size of a distributed source. On the basis of these proofof-principle measurements, the implementation of the proposed method appears feasible.

\section{Technical Challenges to the Implementation}

However, there are still a number of technical challenges to the implementation of the method for application to containerized plutonium assemblies. First, the method does rely upon an assumed energy spectrum of neutrons incident on the detectors. For plutonium assemblies that are only weakly self-attenuating and/or selfmultiplying, the assumption of a spontaneous fission spectrum is probably adequate. For less transparent sources, it may be necessary to first obtain an initial rough estimate of the shape for an assumed

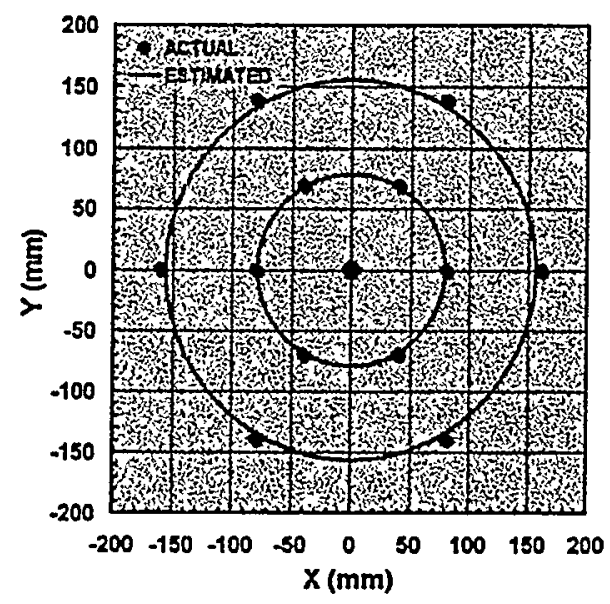

Figure 9. Estimated diameter of each approximate ring source versus actual diameter fission spectrum that can then be corrected using a "quick-and-dirty" Monte Carlo (or maybe even a multi-group diffusion theory) estimate of the actual escape spectrum. Such corrections will probably have to be applied iteratively to incrementally refine sequential estimates of shape and size. However, simple calculational models can be generated automatically during the analysis of the bicovariance to maintain a completely automated measurement system.

A similar difficulty is associated with the effect a container may have on the neutron escape spectrum. Many containers, like the AT400-R, are constructed with a substantial amount of hydrogenous material and thermal neutron absorbers. However, plastic scintillators are essentially insensitive to thermal neutrons, and the hydrogenous material will, to a first order approximation, simply reduce the number of neutrons in the high energy range ( $>1 \mathrm{MeV})$ without significantly changing the basic shape of the spectrum in this range. The proposed method does not rely upon the absolute number of neutrons incident on the detectors but only on the energy spectrum of those neutrons. In other words, the shape estimate for containerized plutonium is measured principally from the uncollided neutron field whose spectrum should remain similar to a fission spectrum in the high energy range. So container effects are expected to induce errors much less significant than those resulting from self-attenuating and/or self-multiplying plutonium assemblies. It may be possible to similarly compensate for these errors using simple automated calculational models. 


\section{Conclusions}

The proposed method to infer the shape and size of plutonium assemblies from passive, stationary NMIS measurements appears feasible. The test measurements performed demonstrated that the method is capable of locating a point source, distinguishing a distributed source from a point source, and estimating the size of a distributed source. In particular, the estimates of the diameters of the two approximate ring sources measured fell within $3 \%$ of the actual diameters. This indicates that the technique may be capable of producing estimates of shape and size of containerized regular shapes of plutonium with accuracy sufficient for transparency or even verification applications. The technique could therefore be of significant utility in both domestic and international safeguards efforts.

\section{References}

[1] J. Hurn, GPS: A Guide to the Next Utility, Trimble Navigation Ltd. (1989).

[2] J. Hurn, Differential GPS Explained: An Expose of the Surprisingly Simple Principles Behind Today's Most Advanced Positioning Technology, Trimble Navigation Ltd. (1993).

[3] J. K. Mattingly, High Order Statistical Signatures from Source-Driven Measurements of Subcritical Fissile Systems, Ph.D. dissertation, University of Tennessee, Knoxville (1998).

[4] C. Wagemans, The Nuclear Fission Process, CRC Press (1991).

[5] G. F. Knoll, Radiation Detection and Measurement, $2^{\text {nd }}$ Ed., Wiley \& Sons (1989).

[6] J. T. Mihalczo, T. E. Valentine, and J. K. Mattingly, "NWIS Methodology," Y/LB-15,953, Oak Ridge Y-12 Plant (1997).

[7] T. E. Valentine, L. G. Chiang, and J. T. Mihalczo, "NMIS for Interrogation of Pu and HEU in AT400-R Containers at Mayak", ORNL/TM-6648 R1, Oak Ridge National Laboratory (1999). 


\section{Distribution:}

Recipients as designated by the author plus:

Y-12 Plant Records Services (3) 9711-5, MS-8169

[Includes 2 copies for OSTI and 1 copy for Central Files]

Note: This distribution page should not be included in the copy of your document (abstract, visuals, article, etc.) submitted to a journal or technical society. 